\title{
Estoque de serapilheira em uma floresta em processo de restauração após mineração de bauxita
}

Litter stock in a forest in process of restoration after bauxite mining

\author{
Kelly de Almeida Silva ${ }^{1,3}$, Sebastião Venâncio Martins ${ }^{1}$, Aurino Miranda Neto ${ }^{1}$ \& Aldo Teixeira Lopes ${ }^{2}$
}

\begin{abstract}
Resumo
O presente estudo teve como objetivo avaliar a serapilheira acumulada em dois ambientes, uma floresta em processo de restauração, após a mineração de bauxita, e um ecossistema de referência (remanescente de floresta secundária em estágio médio de sucessão), e verificar a relação de variáveis ambientais (abertura de dossel, compactação do solo, regeneração natural) com o acúmulo de serapilheira em ambas florestas. Foram alocadas 30 parcelas de $2 \times 2 \mathrm{~m}$ em cada uma das duas florestas. No centro de cada parcela coletou-se todo o material orgânico contido em amostras de $0,5 \times 0,5 \mathrm{~m}$ para posterior obtenção da massa seca. Há dissimilaridade entre as duas florestas, em relação ao estoque de serapilheira e demais variáveis ambientais avaliadas. A floresta em restauração apresentou menor acúmulo de serapilheira que o ecossistema de referência. O maior acúmulo de serapilheira, em ambas florestas, acontece em locais com maior quantidade de indivíduos do estrato de regeneração natural e em locais que apresentam solos menos compactados.

Palavras-chave: abertura do dossel, compactação do solo, ecossistema de referência, regeneração natural.

Abstract

The present study had as objective to evaluate the accumulated litter in two environments, a forest under restoration, after the bauxite mining, and a reference ecosystem (remaining secondary forest in a midsuccessional stage), and investigate the relationship between environmental variables (canopy opening, soil compaction, natural regeneration) and litter accumulation in both forests. Thirty plots of $2 \times 2 \mathrm{~m}$ in each forest were allocated. In the center of each plot, all organic material contained in samples of $0.5 \times 0.5 \mathrm{~m}$ was collected to obtain the dry mass. There is dissimilarity between the two forests, in relation to the litter stock and other environmental variables evaluated. The forest in restoration presents less litter accumulation than the reference ecosystem. The largest litter accumulation in both forests occurs in sites with higher number of individuals in the natural regeneration layer and in sites with lower soil compaction.
\end{abstract}

Key words: canopy openness, soil compaction, reference ecosystem, natural regeneration.

\section{Introdução}

A mineração de bauxita demanda a supressão da vegetação e a remoção dos horizontes superficiais do solo (Grant et al. 2007), sendo necessária a adoção de medidas mitigadoras e compensatórias. Nesse cenário, além da compensação ambiental em áreas próximas do local minerado, torna-se necessária a restauração do ecossistema degradado. A restauração de ambientes alterados pela atividade de mineração requer o retorno da diversidade de espécies e dos processos ecológicos encontrados anteriormente à mineração, além do retorno da resiliência do ecossistema (Norman et al. 2006; Chia et al. 2016). Um dos processos ecológicos que merecem destaque em áreas alteradas em processo de restauração é a produção e acúmulo de serapilheira no piso florestal, que fornece matéria orgânica para o solo e regula a ciclagem de nutrientes nos ecossistemas florestais (Weltzin et al. 2005; Pandey et al. 2007).

\footnotetext{
${ }^{1}$ Universidade Federal de Viçosa, Depto. Engenharia Florestal, Lab. Restauração Florestal, Av. P.H. Rolfs, s/n, Campus UFV, 36570-900, Viçosa, MG, Brasil.

${ }^{2}$ Companhia Brasileira de Alumínio / Votorantim Metais, Setor de Meio Ambiente, Fazenda Chorona s/n, 36790-000, Miraí, MG, Brasil.

${ }^{3}$ Autor para correspondência: kellyalmeidaenf@yahoo.com.br
} 
A serapilheira é constituída em maior escala por material de origem vegetal e em menor escala por material de origem animal (Martins 2016). Ela apresenta entradas e saídas, ou seja, recebe o material (folhas, ramos, frutos, sementes, cascas e flores) por meio da vegetação e o decompõe para suprimento de nutrientes e matéria orgânica para o solo e raízes (Martins 2016).

O estoque de serapilheira no piso florestal é um dos indicadores utilizados para avaliar florestas em processo de restauração (Sperandio et al. 2012; Miranda Neto et al. 2014; Correia et al. 2016; Martins 2016). E, juntamente com o levantamento do estrato de regeneração natural, banco de sementes do solo, chuva de sementes, entre outros, formam os indicadores vegetativos de avaliação de florestas restauradas ou em processo de restauração (Brancalion et al. 2015; Martins 2016). Os indicadores a serem utilizados na avaliação de ecossistemas em restauração devem ser simples e de fácil mensuração (Burton 2014). Os indicadores vegetativos atendem a essa premissa.

Além da avaliação dos indicadores vegetativos individualmente, é interessante verificar a correlação entre os indicadores vegetativos e esses com variáveis ambientais, como abertura de dossel e resistência do solo a penetração. O dossel florestal influencia no controle da quantidade, qualidade e distribuição temporal e espacial da luz, (Jennings et al. 1999), na interceptação das chuvas, na determinação do micro-habitat interno da floresta, afetando a composição florística da comunidade, a produção e decomposição de serapilheira, a invasão de gramíneas agressivas e os processos erosivos (Melo et al. 2007; Martins 2016).

A remoção do solo superficial pela atividade de mineração, apesar do retorno desse material após a extração da bauxita, aumenta o grau de resistência do solo a penetração (compactação do solo). Assim, espera-se que o estoque de serapilheira, associado ao estado físico do solo, em função do nível de compactação, influenciará na sustentação da cobertura vegetal de uma determinada comunidade florestal (Reis 2006).

A avaliação de áreas em restauração é necessária para melhorar as técnicas de restauração e verificar o cumprimento dos objetivos traçados no projeto (Stanturf et al. 2014). Além disso, é importante a avaliação de áreas remanescentes de florestas no entorno da floresta em restauração, que servirão como referência para comparação dos dados coletados entre ambas áreas (Keddy \& Drummond 1996;
Jaunatre et al. 2013; Correia et al. 2016). Neste contexto, o presente estudo teve como objetivo avaliar a serapilheira acumulada de duas florestas, uma em processo de restauração florestal após mineração de bauxita, e outra representada por um remanescente florestal secundário em estágio médio de sucessão (ecossistema de referência); além de analisar variáveis ambientais (abertura do dossel, resistência do solo a penetração e estrato de regeneração) e investigar a relação destas com o acúmulo de serapilheira em ambas florestas.

\section{Material e Métodos}

O presente estudo foi realizado em uma floresta em processo de restauração após mineração de bauxita, via plantio de mudas de espécies arbóreas, Floresta A1, e em um remanescente de floresta secundária preservado em estágio médio de sucessão, representado como ecossistema de referência, Floresta A2.

As florestas estão localizadas no município de São Sebastião da Vargem Alegre (21'4'20"S e $\left.42^{\circ} 38^{\prime} 11^{\prime \prime} W\right)$, estado de Minas Gerais, Sudeste do Brasil, com altitude local variando de 792 a $832 \mathrm{~m}$ (Fig. 1). No entorno dessas florestas, existem áreas de

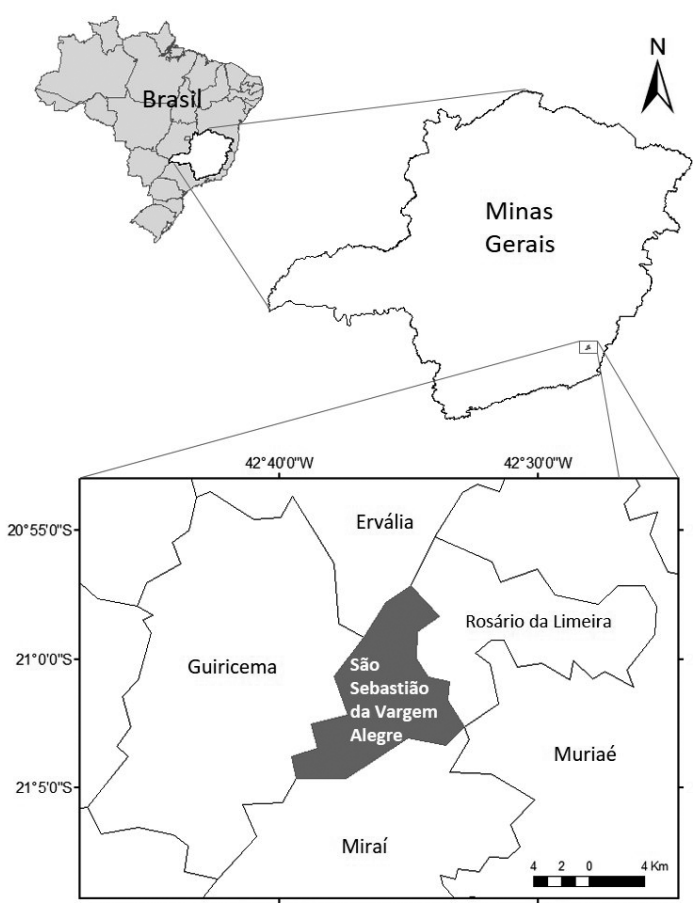

Figura 1 - Localização do município de São Sebastião da Vargem Alegre, Minas Gerais, Brasil.

Figure 1 - Municipality location of São Sebastião da Vargem Alegre, Minas Gerais, Brazil. 
pastagem, fragmentos florestais preservados, plantios de eucalipto e áreas em processo de mineração.

O clima da região, segundo a classificação de Köppen, é do tipo Cwa, clima temperado úmido com inverno seco e verão quente (Sá Júnior et al. 2012). A temperatura média mínima anual é de 18,2 ${ }^{\circ} \mathrm{C}$ e média máxima anual de $31^{\circ} \mathrm{C}$, com temperatura média anual de $23,5{ }^{\circ} \mathrm{C}$ e precipitação média anual de $1.564 \mathrm{~mm}$ (Agevap 2013). A vegetação característica da região é classificada como Floresta Estacional Semidecidual Montana, inserida no Domínio Floresta Atlântica (IBGE 2012).

Na Floresta A1, com 2,18 ha, foi realizada a extração de bauxita, no ano de 2008, pela empresa Companhia Brasileira de Alumínio (CBA)/ Votorantim Metais e, posteriormente, o processo de restauração na área, seguindo as etapas de: recomposição topográfica; deposição da camada fértil de solo (top soil de $0,30 \mathrm{~m}$, retirado e armazenado anteriormente à mineração); correção da acidez do solo e adubação fosfatada; adubação de base (no plantio); plantio heterogêneo de espécies arbóreas (Tab. 1), com espaçamento de $3 \times 2 \mathrm{~m}$; e adubação de cobertura (60 e 120 dias pós-plantio), no entorno das mudas, finalizando as atividades de implantação da restauração no ano de 2010 . A análise da serapilheira acumulada foi realizada cinco anos após o início do processo de restauração da Floresta A1.

A Floresta A2, com 5,30 ha, constitui um trecho remanescente de Floresta Estacional Semidecidual secundário preservado, inserido no Bioma Floresta Atlântica, adjacente à Floresta A1, que se encontra no estágio médio de sucessão, e serviu como ecossistema de referência, auxiliando na avaliação da Floresta A1.

Tabela 1 - Lista de espécies arbóreas utilizadas no plantio da floresta em processo de restauração (Floresta A1), São Sebastião da Vargem Alegre, MG, Brasil.

Table 1 - List of tree species used in planting forest in restoration process (Forest A1), São Sebastião da Vargem Alegre, MG, Brazil.

\begin{tabular}{|c|c|}
\hline Família & Espécie \\
\hline \multirow[t]{2}{*}{ Anacardiaceae } & Schinus terebinthifolius Raddi \\
\hline & Tapirira guianensis Aubl. \\
\hline Apocynaceae & Tabernaemontana laeta Mart. \\
\hline \multirow[t]{2}{*}{ Bignoniaceae } & Jacaranda puberula Cham. \\
\hline & Sparattosperma leucanthum (Vell.) K.Schum. \\
\hline Bixaceae & Bixa orellana L. \\
\hline Boraginaceae & Cordia trichotoma (Vell.) Arráb. ex. Steud. \\
\hline Cannabaceae & Trema micrantha (L.) Blume \\
\hline Caricaceae & Jacaratia spinosia (Aubl.) A.DC. \\
\hline \multirow[t]{2}{*}{ Euphorbiaceae } & Croton floribundus Spreng. \\
\hline & Joannesia princeps Vell. \\
\hline \multirow[t]{10}{*}{ Fabaceae } & Anadenanthera macrocarpa (Benth.) Brenan \\
\hline & Andira anthelmia (Vell.) Benth. \\
\hline & Apuleia leiocarpa (Vogel) J.F.Macbr. \\
\hline & Enterolobium contortisiliquum (Vell.) Morong \\
\hline & Erythrina falcata Benth. \\
\hline & Hymenaea courbaril L. \\
\hline & Inga edulis Mart. \\
\hline & Leucaena leucocephala (Lam.) de Wit* \\
\hline & Machaerium nyctitans (Vell.) Benth. \\
\hline & Peltophorum dubium (Spreng.) Taub. \\
\hline
\end{tabular}




\begin{tabular}{|c|c|}
\hline Família & Espécie \\
\hline & Piptadenia gonoacantha (Mart.) J.F.Macbr. \\
\hline & Schizolobium parahyba (Vell.) Blake \\
\hline & Senna multijuga (Rich.) H.S.Irwin \& Barneby \\
\hline Lamiaceae & Aegiphila integrifolia (Jacq.) Moldenke \\
\hline Lauraceae & Aniba firmula (Nees \& Mart.) Mez \\
\hline \multirow[t]{2}{*}{ Malvaceae } & Ceiba speciosa (A.St.-Hil.) Ravenna \\
\hline & Luehea divaricata Mart. \& Zucc. \\
\hline Melastomataceae & Tibouchina granulosa (Desr.) Cogn. \\
\hline \multirow[t]{4}{*}{ Meliaceae } & Cabralea canjerana (Vell.) Mart. \\
\hline & Cedrela fissilis Vell. \\
\hline & Guarea guidonia (L.) Sleumer \\
\hline & Melia azedarach L. * \\
\hline \multirow[t]{2}{*}{ Moraceae } & Artocarpus heterophyllus Lam.* \\
\hline & Morus nigra L. * \\
\hline \multirow[t]{2}{*}{ Myrtaceae } & Syzygium cumini (L.) Skeels * \\
\hline & Eucalyptus sp. * \\
\hline \multirow[t]{2}{*}{ Rosaceae } & Cydonia oblonga Mill. * \\
\hline & Eriobotrya japonica (Thunb.) Lindl. * \\
\hline Rubiaceae & Genipa americana $\mathrm{L}$. \\
\hline Sapindaceae & Sapindus saponaria L. \\
\hline \multirow[t]{3}{*}{ Solanaceae } & Solanum bullatum Vell. \\
\hline & Solanum mauritianum Scop. \\
\hline & Solanum paniculatum L. \\
\hline Vochysiaceae & Callisthene fasciculata Mart. \\
\hline
\end{tabular}

* Espécies exóticas no Brasil.

Foram alocadas 30 parcelas de $2 \times 2 \mathrm{~m}$ em cada uma das florestas estudadas (Floresta A1 e Floresta A2), distribuídas em seis linhas de cinco parcelas, com distância de $5 \mathrm{~m}$ entre parcelas, na linha, e de $40 \mathrm{~m}$ entre linhas (Fig. 2).

Para análise da serapilheira acumulada, no centro de cada uma das 60 parcelas (30 parcelas na Floresta A1 e 30 parcelas na Floresta A2) foi utilizado um gabarito de $0,5 \times 0,5 \mathrm{~m}\left(0,25 \mathrm{~m}^{2}\right)$ para a coleta de todo o material orgânico (folhas, ramos, frutos e flores) contido no interior do gabarito, sendo a coleta realizada na estação seca. Posteriormente, esse material foi embalado em sacos plásticos, devidamente identificados, e levado para o Laboratório de Restauração Florestal (LARF) da Universidade Federal de Viçosa (UFV), Viçosa, MG, onde foi transferido para sacos de papel, com a identificação de cada parcela pertencentes às

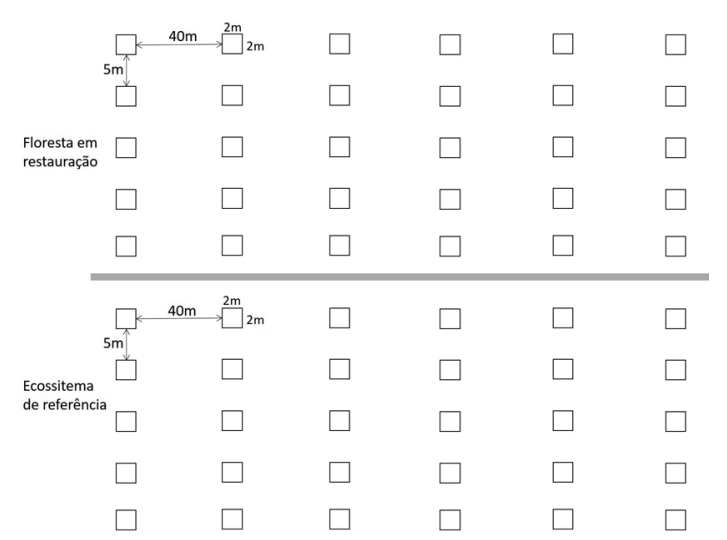

Figura 2 - Distribuição das parcelas na floresta em restauração e no ecossistema de referência, São Sebastião da Vargem Alegre, MG, Brasil.

Figure 2 - Distribution of plots in restoration forest and reference ecosystem, São Sebastião da Vargem Alegre, MG, Brazil. 
Florestas A1 e A2, e colocados em estufa a $70^{\circ} \mathrm{C}$ durante 72 horas. Após a secagem, o material foi pesado em balança analítica de precisão para obtenção da massa seca em gramas.

A quantidade de serapilheira acumulada, encontrada nas duas florestas (Floresta A1 e Floresta A2), foi estimada para kg.ha- ${ }^{-1}$ e as médias obtidas foram comparadas entre ambas através do teste $t$ de Student para amostras independentes com o auxílio do software Statistica 10 (Statsoft 2011). Da mesma forma, foram obtidas as médias de indivíduos presentes na regeneração natural (indivíduos. $\mathrm{m}^{-2}$ ), abertura do dossel (\%) e compactação do solo $\left(\mathrm{kg} . \mathrm{cm}^{-2}\right)$ para comparação entre as duas florestas através do teste $t$ de Student para amostras independentes com o auxílio do software Statistica 10 (Statsoft 2011).

Os dados de serapilheira acumulada, abertura de dossel, indivíduos presentes na regeneração natural e compactação de solo também foram utilizados para verificar a similaridade entre as duas florestas através de análise de agrupamento. Assim, foi empregado o coeficiente Distância de Manhattan, a partir de uma matriz de dados quantitativos. Para interpretar a similaridade, foi utilizado o método de agrupamento Ligação Completa, gerando o valor de correlação cofenética e produzindo um dendrograma em que as amostras semelhantes (30 parcelas da Floresta A1 e 30 parcelas da Floreta A2), de acordo as variáveis escolhidas, foram agrupadas entre si. As análises foram efetuadas no software Fitopac 2.1 (Shepherd 2010).

Calculou-se a matriz de correlação de Pearson entre os valores de serapilheira acumulada, indivíduos presentes no estrato de regeneração natural, resistência do solo a penetração e abertura do dossel com o auxílio do software Statistica 10 (Statsoft 2011). Esta análise foi realizada com o intuito de avaliar a relação da serapilheira com as variáveis ambientais.

Para a análise da regeneração natural, todos os indivíduos arbustivo-arbóreos com altura $\geq 0,30$ $\mathrm{m}$ e diâmetro à altura do peito $(\mathrm{DAP}=1,30 \mathrm{~m}) \leq$ $5,0 \mathrm{~cm}$, presentes nas parcelas demarcadas, foram contabilizados.

A abertura do dossel foi determinada por fotografia digital hemisférica, no centro de cada parcela da Floresta A1 e Floresta A2, obtida com uma lente de $8 \mathrm{~mm}$, que fornece um campo de visão de $180^{\circ}$, a qual foi acoplada a uma câmera fotográfica digital fixada em um tripé de cabeça móvel regulável e com bolha niveladora para estabilizar a câmera. Posteriormente, as fotografias foram processadas no Programa Gap Light Analyzer 2.0 (Frazer et al. 1999).

Para a determinação da compactação do solo foi utilizado um penetrômetro digital de impacto modelo Penetrolog PLG1020 Falker ${ }^{\circledR}$. A mensuração foi feita coletando-se dois pontos em cada parcela (60 parcelas), até a profundidade de 40 $\mathrm{cm}$, sendo utilizado a média dos dois valores mais elevados da pressão atingida, em cada parcela, na profundidade de 0 a $40 \mathrm{~cm}$.

\section{Resultados}

A serapilheira média acumulada no piso florestal da floresta em processo de restauração há cinco anos (Floresta A1) foi de $4.640 \pm 1.812$ kg.ha- ${ }^{-1}$, enquanto no ecossistema de referência (Floresta A2) este valor foi de $6.339 \pm 1.357$ kg.ha ${ }^{-1}$, evidenciando diferença significativa entre as duas florestas $(p<0,05)$. Para as demais variáves avaliadas também houve diferença significativa $(p<0,05)$ entre as duas florestas avaliadas, como pode-se constatar: número de indivíduos presentes na regeneração natural da Floresta A1 foi de 0,51 $\pm 0,45$ indivíduos. $\mathrm{m}^{-2}$ e na Floresta $\mathrm{A} 2$ foi de 2,62 $\pm 1,10$ indivíduos. $\mathrm{m}^{-2}$; para a resistência do solo a penetração o valor médio foi de $37,98 \pm 6,10 \mathrm{~kg} \cdot \mathrm{cm}^{-2}$ na Floresta $\mathrm{A} 1$ e $17,01 \pm 3,56 \mathrm{~kg} . \mathrm{cm}^{-2}$ na Floresta A2; e verificou-se o valor médio de $28,59 \pm 7,41 \%$ de abertura do dossel na Floresta A1 e 19,07 \pm $5,76 \%$ na Floresta A2.

As análises de correlação de Pearson mostraram que a serapilheira acumulada apresentou correlação significativa $(p<0,05)$ positiva com o número de indivíduos arbustivo-arbóreos presentes na regeneração e negativa com a resistência do solo a penetração. Constatou-se também correlação significativa $(p<0,05)$ negativa entre o número de indivíduos arbustivo-arbóreos e a resistência do solo a penetração, bem como entre o número de indivíduos arbustivo-arbóreos e a abertura do dossel. Em relação à abertura do dossel e resistência do solo a penetração, houve diferença significativa positiva $(p<0,05)$ (Tab. 2; Fig. 3a-e).

A análise de agrupamento também mostrou a dissimilaridade entre as duas florestas, em relação ao estoque de serapilheira e demais variáveis ambientais avaliadas, com uma correlação cofenética de 0,60. O dendrograma gerado mostra a dicotomia entre as duas florestas, formando dois grupos, um com o predomínio de parcelas correspondentes a Floresta A1 e outro grupo com o predomínio de parcelas correspondentes a Floresta A2 (Fig. 4). 
Tabela 2 - Correlação de Pearson entre serapilheira acumulada e variáveis ambientais, para o total das 60 parcelas na floresta em processo de restauração e ecossistema de referência, São Sebastião da Vargem Alegre, MG, Brasil. Valores com asterisco indicam correlação.

Table 2 - Pearson correlation between accumulated litter and environmental variables, to total 60 plots in forest restoration process and reference ecosystem, São Sebastião da Vargem Alegre, MG, Brazil. Values with an asterisk indicate correlation.

\begin{tabular}{lcccc}
\hline & Serapilheira & $\begin{array}{c}\text { Número de } \\
\text { indivíduos }\end{array}$ & $\begin{array}{c}\text { Resistência do } \\
\text { solo a penetração }\end{array}$ & $\begin{array}{c}\text { Abertura do } \\
\text { dossel }\end{array}$ \\
\hline Serapilheira & 1,0000 & - & - & - \\
Número de indivíduos & $0,4911^{*}$ & 1,0000 & - & - \\
Resistência do solo a penetração & $-0,3106^{*}$ & $-0,7252^{*}$ & 1,0000 & - \\
Abertura do dossel & $-0,1614$ & $-0,4388^{*}$ & $0,5759^{*}$ & 1,0000 \\
\hline
\end{tabular}

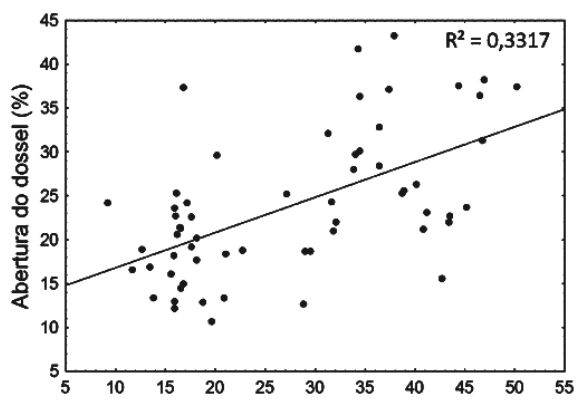

Resistência do solo a penetração $\left(\mathrm{kg} \mathrm{cm}^{-2}\right)$ a

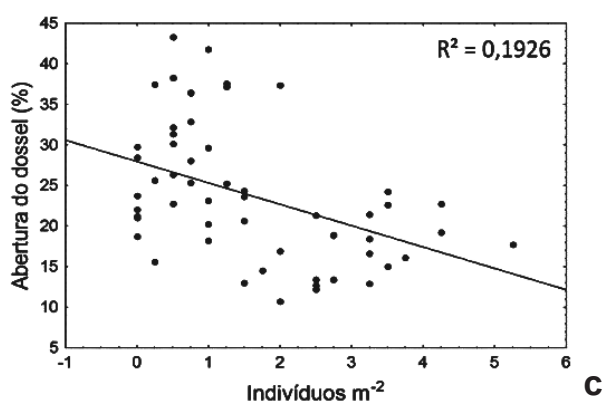

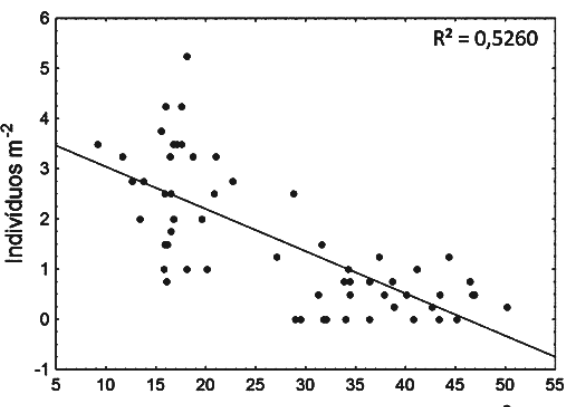

Resistência do solo a penetração $\left(\mathrm{kg} \mathrm{cm}^{-2}\right)$

b

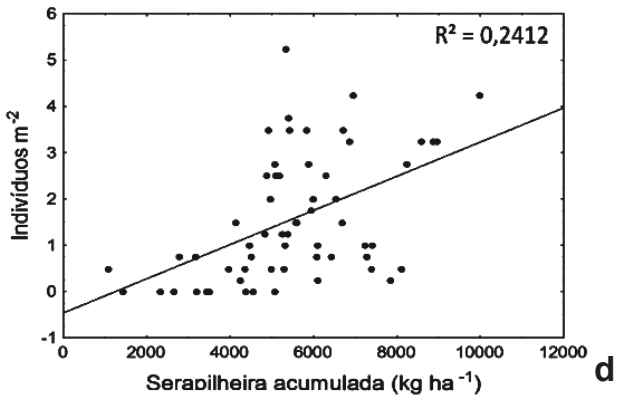

$R^{2}=0,0965$

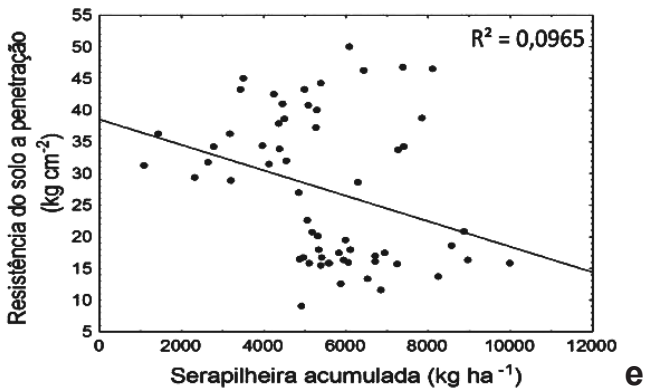

Figura 3 - a-e. Correlação entre abertura do dossel (\%) e resistência do solo à penetração $\left(\mathrm{kg} . \mathrm{cm}^{-2}\right)$ - a. número de indivíduos presentes na regeneração natural $\left(\right.$ ind. $\left.\mathrm{m}^{-2}\right)$ e resistência do solo à penetração $\left(\mathrm{kg} . \mathrm{cm}^{-2}\right)$; b. abertura do dossel $(\%)$ e número de indivíduos presentes na regeneração natural (ind. $\mathrm{m}^{-2}$ ); c. número de indivíduos presentes na regeneração natural (ind. $\mathrm{m}^{-2}$ ) e serapilheira acumulada $\left(\mathrm{kg} \cdot \mathrm{ha}^{-1}\right)$; d. resistência do solo à penetração $\left(\mathrm{kg} \cdot \mathrm{cm}^{-2}\right)$ e serapilheira acumulada $\left(\mathrm{kg} \cdot \mathrm{ha}^{-1}\right)$; e.para o total das 60 parcelas na floresta em processo de restauração e no ecossistema de referência, São Sebastião da Vargem Alegre, MG, Brasil. Figure 3 - a-e. Correlation between canopy openness (\%) and soil resistance to penetration $\left(\mathrm{kg} \cdot \mathrm{cm}^{-2}\right)$ - a. number of individuals present in the natural regeneration (ind. $\left.\mathrm{m}^{-2}\right)$ and soil resistance to penetration $\left(\mathrm{kg} . \mathrm{cm}^{-2}\right)$; b. canopy openness $(\%)$ and number of individuals present in the natural regeneration (ind. $\left.\mathrm{m}^{-2}\right)$; c. number of individuals present in the natural regeneration (ind. $\mathrm{m}^{-2}$ ) and accumulated litter $\left(\mathrm{kg} \cdot \mathrm{ha} \mathrm{a}^{-1}\right) ; \mathrm{d}$. soil resistance to penetration $\left(\mathrm{kg} . \mathrm{cm}^{-2}\right)$ and accumulated litter $\left(\mathrm{kg}_{\mathrm{gha}} \mathrm{h}^{-1}\right)$; e. for the total of 60 plots in forest restoration process and ecosystem reference, São Sebastião da Vargem Alegre, MG, Brazil. 


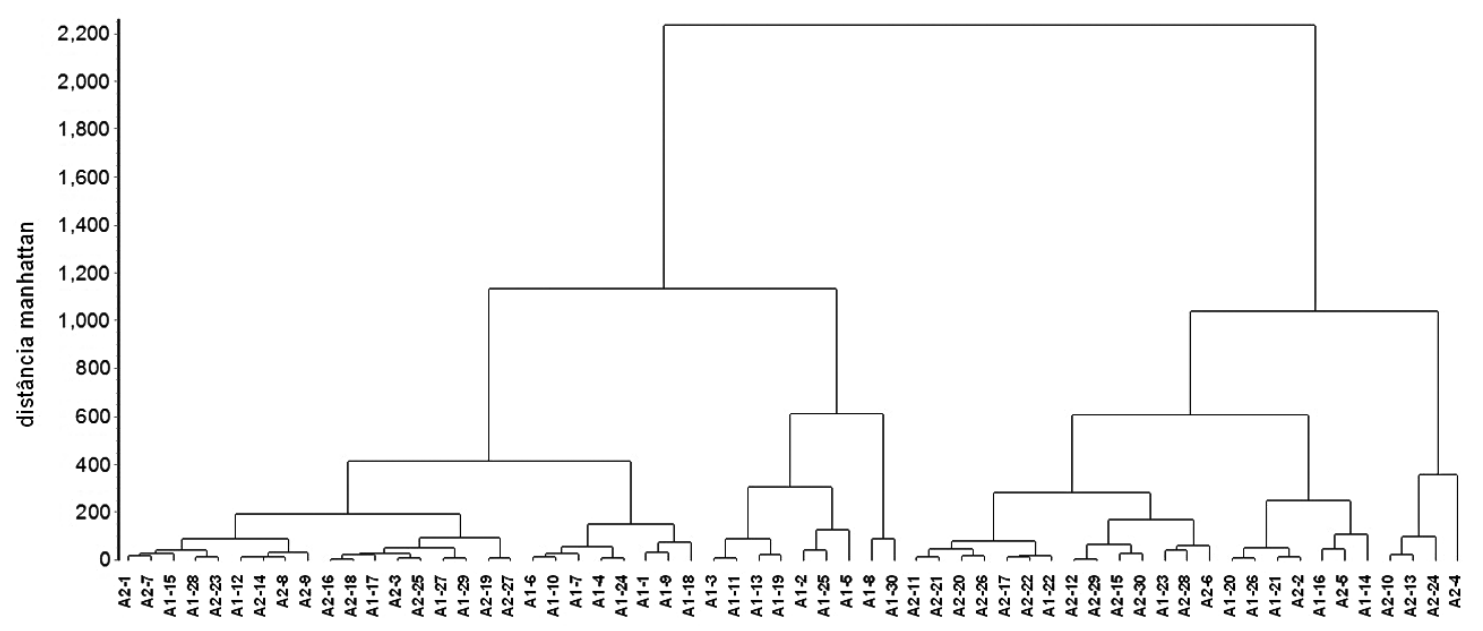

Figura 4 - Dendrograma da similaridade gerado pelo método de Ligação Completa, empregando o coeficiente Distância de Manhattan, a partir de uma matriz de dados quantitativos das variáveis ambientais (serapilheira acumulada, abertura de dossel, indivíduos presentes na regeneração natural e compactação de solo) entre a Floresta A1 (parcelas A1-1 até A1-30) e Floresta A2 (parcelas A2-1 até A2-30), São Sebastião da Vargem Alegre, MG, Brasil.

Figure 4 - Dendrogram of the similarity generated by the Complete Linkage method, using the Manhattan Distance coefficient, from a quantitative data matrix of the environmental attributes (accumulated litter, canopy opening, present in natural regeneration and soil compaction) between Forest A1 (plots A1-1 to A1-30) and Forest A2 (plots A2-1 to A2-30), São Sebastião da Vargem Alegre, MG, Brazil.

\section{Discussão}

A menor quantidade de serapilheira acumulada na Floresta A1 (4.640 kg.ha- $\left.{ }^{-1}\right)$ quando comparada com a Floresta A2 (6.339 kg.ha- $\left.{ }^{-1}\right)$ pode ser explicada pelo fato da Floresta A2 tratar-se de um remanescente florestal bem conservado em estágio médio de sucessão enquanto que a Floresta A1 encontra-se em processo de restauração há apenas cinco anos. Entretanto, a Floresta A1 apresentou um estoque de serapilheira superior ao encontrado em estudos com florestas em restauração com maiores idades: $3.177 \mathrm{~kg} \cdot \mathrm{ha}^{-1} \mathrm{em}$ floresta em processo de restauração há 23 anos, em domínio de Floresta Ombrófila Densa de Terras Baixas (Correia et al. 2016); e 3.432 kg.ha- em floresta restaurada com idade de 40 anos, em Floresta Estacional Semidecidual (Miranda Neto et al. 2014). Contudo, o valor encontrado no presente estudo mostrou-se inferior ao encontrado por Sperandio et al. (2012) que constataram 5.670 kg.ha ${ }^{-1}$, para uma floresta restaurada com espécies tropicais diversas com idade de 18 anos, em região de Floresta Estacional Semidecidual.

Os dados mostraram que apesar da floresta em restauração ainda ser nova (cinco anos), já apresenta um bom aporte de serapilheira acumulada, possivelmente influenciada pelo desenvolvimento dos indivíduos plantados na área e pelo ecossistema de referência, que se encontra adjacente à floresta em restauração, contribuindo com a dispersão de propágulos vegetativos e reprodutivos.

A dissimilaridade entre a floresta em processo de restauração e o ecossistema de referência pode ser explicado em função das distintas características de formação e composição de ambas florestas, mas principalmente, devido a diferença de idade e, ou estágio sucessional, o que é reflexo da diferença nos valores de estoque de serapilheira, compactação do solo e abertura do dossel. Outro fator que possivelmente ajuda na distinção entre as florestas é que o estrato de regeneração natural da Floresta A1 se encontra em fase de construção e ainda necessita de propágulos oriundos de fontes externas e das árvores introduzidas no plantio que ainda não atingiram o período reprodutivo e de frutificação. Além disso, como a Floresta A1 é uma área que sofreu alteração na conformidade e estrutura do terreno em virtude da atividade de mineração, já é esperado que apresente um solo com maior grau de compactação em relação a Floresta A2.

Em solos mais compactados, neste caso, em função da mineração de bauxita, sementes de muitas espécies encontram dificuldade em germinar, se desenvolver e propagar, e isso pode refletir na composição e quantidade de indivíduos estabelecidos nestes ambientes (Chia et al. 2016). 
O conhecimento do nível de abertura do dossel permite inferir sobre as condições de luz no interior da floresta (Trichon et al. 1998; Martins \& Rodrigues 2002) e, consequentemente, na presença de espécies mais ou menos tolerantes ao sombreamento proporcionado pelo dossel (Pinto et al. 2008), bem como sobre o estágio sucessional em que um ecossistema se encontra. É o caso das florestas estudadas, em que a Floresta A1, que possui um dossel mais aberto, permite maior entrada de luz, o desenvolvimento principalmente de espécies pioneiras e secundárias iniciais e se encontra, portanto, em estágio inicial de sucessão. Enquanto que a Floresta A2, que possui dossel mais fechado, permite menor entrada de luz, o desenvolvimento principalmente de espécies tardias e se encontra, portanto, em estágio médio de sucessão.

As análises de correlação entre a serapilheira acumulada e as variáveis ambientais mostraram que locais com maior número de indivíduos da regeneração natural e menor compactação do solo apresentam maior acúmulo de serapilheira. Assim como a abertura do dossel é maior em locais com solo mais compactado e que, portanto, se verifica menor presença de indivíduos regenerantes. Já em locais com dossel mais fechado há maior presença de regeneração natural.

$\mathrm{O}$ aumento do estoque de serapilheira pode elevar o acúmulo de matéria orgânica fresca na superfície do solo (Norby et al. 2001; Sayer et al. 2006) e promover a proliferação de raízes nas camadas superficiais do piso florestal, a depender da disponibilidade de nutrientes na serapilheira (Sayer et al. 2006). Esse aumento de raízes pode promover um solo menos compactado.

A densidade de indivíduos vegetais e sua composição, além do comportamento variado de deciduidade das espécies florestais, podem influenciar diretamente a quantidade de serapilheira depositada no piso da floresta (Vogt et al. 1986; Machado et al. 2008). Normalmente, espera-se obter maior quantidade de serapilheira quanto maior for o número de indivíduos.

Estudo realizado em uma área em restauração junto com outra área adjacente de Floresta Ombrófila Densa primária, no Norte do Espírito Santo, também foi constatado menor compactação do solo em ambientes com dossel mais fechado (Correia et al. 2016). Essa relação acontece porque a maior cobertura do dossel pode minimizar o impacto das gotas de chuva, reduzir a temperatura no solo em função da redução do nível de luminosidade, aumentar a ciclagem de nutrientes (Sperandio et al.
2012), e aumentar a umidade no solo e no sub-bosque (Correia et al. 2016).

A abertura do dossel pode influenciar a maior ou menor presença de espécies florestais e animais no estrato de regeneração natural, sendo que ambientes com maior cobertura do dossel pode proporcionar proteção às plântulas e habitats mais adequados para animais dispersores (Tewksburry \& Lloyd 2001; Dias et al. 2005).

\section{Conclusões}

A floresta em processo de restauração apresentou menor acúmulo de serapilheira que o ecossistema de referência (remanescente florestal adjacente).

O maior acúmulo de serapilheira em ambas florestas aconteceu em locais com maior quantidade de indivíduos do estrato de regeneração natural e em locais que apresentaram solos menos compactados.

\section{Agradecimentos}

Ao Conselho Nacional de Desenvolvimento Científico e Tecnológico (CNPq), a concessão da bolsa de Doutorado à primeira autora, e de Produtividade em Pesquisa ao segundo autor; e à Companhia Brasileira de Alumínio / Votorantim Metais, o financiamento do projeto.

\section{Referências}

AGEVAP - Associação Pró-Gestão das Águas da Bacia Hidrográfica do Rio Paraíba do Sul (2013) Plano municipal de saneamento básico, São Sebastião da Vargem Alegre, MG. AGEVAP, Resende. 234p.

Brancalion PHS, Gandolfi S \& Rodrigues RR (2015) Restauração florestal. Oficina de Textos, São Paulo. 432p.

Burton PJ (2014) Considerations for monitoring and evaluating forest restoration. Journal of Sustainable Forestry 33: S149-S160.

Chia KA, Koch JM, Sadler R \& Turner SR (2016) Reestablishing the mid-storey tree Persoonia longifolia (Proteaceae) in restored forest following bauxite mining in southern Western Australia. Ecological Research 31: 627-638.

Correia GGS, Martins SVM, Miranda Neto A \& Silva KA (2016) Estoque de serapilheira em floresta em restauração e em Floresta Atlântica de Tabuleiro no Sudeste brasileiro. Revista Árvore 40: 13-20.

Dias ATC, Zaluar HLT, Ganade G \& Scarano FR (2005) Canopy composition influencing patch dynamics in a Brazilian sandy coastal plain. Journal of Tropical Ecology 21: 343-347.

Frazer GW, Canham CD \& Lertzman KP (1999) Gap Light Analyzer (GLA): imaging software to extract canopy structure and gap light transmission indices from 
true-colour fisheye photographs, users manual and program documentation. Simon Fraser University, Burnaby, Institute of Ecosystem Studies, New York. $36 \mathrm{p}$.

Grant CD, Ward SC \& Morley SC (2007) Return of ecosystem function to restored bauxite mines in western Australia. Restoration Ecology 15: S94-S103.

IBGE - Instituto Brasileiro de Geografia e Estatística (2012) Manual técnico da vegetação brasileira. $2^{\mathrm{a}}$ ed. Instituto Brasileiro de Geografia e Estatística, Rio de Janeiro. 275p.

Jaunatre R, Buisson E, Muller I, Morlon H, Mesleard F \& Dutoit T (2013) New synthetic indicators to assess community resilience and restoration success. Ecological Indicators 29: 468-477.

Jennings SB, Brown ND \& Sheil D (1999) Assessing forest canopies and understorey illumination: canopy closure, canopy cover and oyther measures. Forestry 72: 59-73.

Keddy PA \& Drummond CG (1996) Ecological properties for the evaluation, management, and restoration of temperate deciduous forest ecosystems. Ecological Applications 6: 748-762.

Machado MR, Piña-Rodrigues FCM \& Pereira MG (2008) Produção de serapilheira como bioindicador de recuperação em plantio adensado de revegetação. Revista Árvore 32: 143-151.

Martins SV \& Rodrigues RR (2002) Gap-phase regeneration in a semideciduous mesophytic forest, southeastern Brazil. Plant Ecology 163: 51-62.

Martins SV (2016) Recuperação de áreas degradadas: ações em áreas de preservação permanente, voçorocas, taludes rodoviários e de mineração. $4^{\mathrm{a}}$ ed. Aprenda Fácil, Viçosa. 270p.

Melo ACG, Miranda DLC \& Durigan G (2007) Cobertura de copas como indicador de desenvolvimento estrutural de reflorestamentos de restauração de matas ciliares no médio vale do Paranapanema, SP, Brasil. Revista Árvore 31: 321-328.

Miranda Neto A, Martins SV, Silva KA \& Gleriani JM (2014) Banco de sementes do solo e serapilheira acumulada em floresta restaurada. Revista Árvore 38: 609-620.

Norby RJ, Cotrufo MF, Ineson P, O’Neill EG \& Canadell JG (2001) Elevated $\mathrm{CO}_{2}$, litter chemistry and decomposition: a synthesis. Oecologia 127: 153-165.

Norman MA, Koch JM, Grant CD, Morald TK \& Ward SC (2006) Vegetation succession after bauxite mining in western Australia. Restoration Ecology 14: $278-288$.
Pandey RR, Sharma G, Tripathi SK \& Singh AK (2007) Litterfall, litter decomposition and nutrient dynamics in a subtropical natural oak forest and managed plantation in northeastern India. Forest Ecology and Management 240: 96-104.

Pinto SIC, Martins SV, Barros NF, Dias HCT \& Kunz SH (2008) Influence of environmental variables on the shrub and tree species distribution in two Semideciduous Forest sites in Viçosa, Minas Gerais, Brazil. Revista de Biología Tropical 56: 1557-1569.

Reis LL (2006) Monitoramento da recuperação ambiental de áreas de mineração de bauxita na Floresta Nacional de Saracá-Taquera, Porto Trombetas (PA). Tese de Doutorado. Universidade Federal Rural do Rio de Janeiro, Seropédica. 159p.

Sá Júnior A, Carvalho LG, Silva FF \& Alves NC (2012) Applicacion of the Köppen classification for climatic zoning in the state of Minas Gerais, Brazil. Theoretical and Applied Climatology 108: 1-7.

Sayer EJ, Tanner EVJ \& Cheesman AW (2006) Increased litterfall changes fine root distribution in a moist tropical forest. Plant and Soil 281: 5-13.

Shepherd GJ (2010) Fitopac 2.1. Departamento de Botânica, Universidade Estadual de Campinas, Campinas. Disponível em $<$ https://pedroeisenlohr.webnode.com. br/fitopac/>. Acesso em 12 Setembro 2016.

Sperandio HV, Cecílio RA, Silva VH, Leal GF, Brinate IB \& Caldeira MVW (2012) Emprego da serapilheira acumulada na avaliação de sistemas de restauração florestal em Alegre-ES. Floresta e Ambiente 19: 460-467.

StanturfJA, PalikBJ \& Dumroese RK (2014) Contemporary forest restoration: a review emphasizing function. Forest Ecology and Management 331: 292-323.

Statsoft (2011) Statistica data analysis software system. Version 10. Tulsa.

Tewksburry JJ \& Lloyd JD (2001) Positive interactions under nurse-plants: spatial scale, stress gradients and benefactor size. Oecologia 127: 425-434.

Trichon V, Walter JMN \& Laumonier Y (1998) Identifying spatial patterns in the tropical rain forest structure using hemispherical photographs. Plant Ecology 137: 227-244.

Vogt KA, Grier CC \& Vogt DJ (1986) Production, turnover, and nutrient dynamics of above- and belowground detritus of world forests. Advances in Ecological Research 15: 303-377.

Weltzin JF, Keller JK, Bridgham SD, Paster J, Allen BP \& Chen J (2005) Litter controls plant community composition in a northern fen. Oikos 110: 537-546. 